\title{
Self-tuning of cosmological constant and exit from inflation *
}

\author{
Jihn E. Kim \\ School of Physics, Seoul National University, Seoul 151-747, Korea \\ jekim@phyp.snu.ac.kr
}

\begin{abstract}
I review the recent 5D self-tuning solutions of the cosmological constant problem, and try to unify two cosmological constant problems within the framework of the self-tuning solutions. One problem, the large cosmological constant needed for inflation, is interpreted by starting with the parameters allowing only the $\mathrm{dS}$ vacuum, and the vanishing cosmological constant at a true vacuum is realized by changing parameters by exit from inflation at the brane such that the self-tuning solution is allowed.
\end{abstract}

Keywords: brane; self-tuning of cosmological constant; brane inflation.

PACS Nos.: 98.80.Es, 98.80.C, 12.25.Mj

\section{Introduction}

In particle physics, there are three vacuum energies to deal with: (i) the GUT scale vacuum energy for inflation, (ii) the vanishing cosmological constant at the minimum which is theoretically favored, and (iii) the observed tiny vacuum energy $(0.003 \mathrm{eV})^{4}$ at $z=10-100$. The tiny vacuum energy discovered from Type $1 \mathrm{~A}$ supernova data and confirmed by the WMAP data makes the cosmological constant problem more difficult. Here, we do not discuss this tiny vacuum energy. Simply, we assume that somehow this tiny vacuum energy is resolved by a (pseudo)scalar field 1 whose misalignment shifts the vacuum energy a little bit from a true minimum of the vanishing vacuum energy with $\omega=p / \rho \leq 0.8$. The cosmological $\operatorname{constant}(\Lambda=$ $\left.8 \pi G_{N} V_{0}\right)$ is a term in Einstein's equation

$$
R_{\mu \nu}-\frac{1}{2} R g_{\mu \nu}-8 \pi G_{N} V_{0} g_{\mu \nu}=8 \pi G_{N} T_{\mu \nu} .
$$

The LHS of (11) without $\Lambda$ vanishes when the spacetime is flat, $g_{\mu \nu}=\eta_{\mu \nu}$. Thus, if matter is present, the RHS of (11) is nonvanishing and hence spacetime is not flat, i.e. $g_{\mu \nu} \neq \eta_{\mu \nu}$. Therefore, under a reasonable assumptions, Eq. (11) must lead to an evolving universe. In 1910's, the universe looked as if it were a static one. So, Einstein introduced a compensating term, $\Lambda g_{\mu \nu}$, on the LHS of (11) to make the solution static. This is the birth of the cosmological constant. In this talk, I will neglect matter(i.e. quantum excitations), and hence we are looking for the vacuum

*Talk presented at 2003 Int. Symposium on Cosmology and Particle Physics(CosPA-03), National Taiwan Univ., Taiwan, Nov. 13-15, 2003. 
solution. Without matter but with a nonvanishing cosmological constant $\Lambda$, it is not possible to have a static solution.

Probably an easy way to see the cosmological constant(c.c.) problem is to start with an action where it is easy to find out possible symmetries. The LHS of the Einstein equation with the c.c. is obtained from the following action

$$
S=\int d^{4} x \sqrt{-g}\left(\frac{M^{2}}{2} R-V_{0}\right)
$$

where $g$ is the determinant of the metric tensor, $R$ is the Ricci scalar, $M^{2}$ is the reduced Planck mass $M^{2}=1 / 8 \pi G_{N}$, and $V_{0}$ is a constant which is related to the cosmological constant by $V_{0}=M^{2} \Lambda$.

The difficulty with the c.c. problem is that there is no symmetry working for it to vanish. An obvious symmetry is the scale invariance, but it is badly broken. At the electroweak scale, we need a mass parameter of order $100 \mathrm{GeV}$, so the c.c. is expected to be $10^{56}$ times larger than the current value. This cosmological constant problem surfaced as a very severe one when the spontaneous symmetry breaking of the electroweak theory was extensively discussed 2 .

The Einstein equation can be solved with an appropriate ansatz for the metric. For example, a flat space ansatz is $g_{\mu \nu}=\eta_{\mu \nu}$, and a de $\operatorname{Sitter}(\mathrm{dS})$ space ansatz is

$$
d s^{2}=-d t^{2}+e^{2 \sqrt{\Lambda} t} d \mathbf{x}^{2}
$$

In $4 \mathrm{D}$, the flat space is possible with the vanishing vacuum energy. Thus, to have a flat space one has to fine-tune the parameters in the action such that the vacuum energy is exactly zero. To have a flat $4 \mathrm{D}$ space with a finite range of parameters in the action, one must go beyond 4D.

Our 4D may come from higher dimensions. In this regard, the RandallSundrum(RS) type models 3 are very interesting. These are most easily studied in $5 \mathrm{D}$. It will be sufficient if we, the $4 \mathrm{D}$ observers, see the vanishing effective $4 \mathrm{D}$ c.c. even though we start with nonzero $5 \mathrm{D}$ c.c. Indeed, the RS-II 3 model starts with a negative $5 \mathrm{D}$ c.c. $\Lambda_{b}$ (bulk c.c.), $A d S_{5}$, and a 3 -brane(s) with brane tension $\Lambda_{1}$, but allow flat $4 \mathrm{D}$, as shown by the line element

$$
d s^{2}=\beta^{2}(y)\left(-d t^{2}+d \mathbf{x}^{2}\right)+d y^{2}
$$

where $\beta(y)$ is the warp factor. If there exists a reasonable solution with the line element (4), then it describes a flat space. It is usually assumed that matter is present at the brane $\mathrm{B} 1$ located at $y=0$. Indeed, a flat $4 \mathrm{D}$ is possible with one fine-tuning, $k_{b}=k_{1}$, with the exponentially suppressed warp factor for large $y$; thus the 5 th direction is not noticeable to the 4D observer of $\mathrm{B} 1$ and there can results a consistent $4 \mathrm{D}$. An important thing to note is that a flat $4 \mathrm{D}$ is obtained, starting with nonzero c.c. But still a fine-tuning between parameters is needed. 


\section{Self-tuning solutions}

Strong self-tuning solution: Without fine-tuning there exists a flat-space solution which is not continuously connected to dS or AdS solutions. It was tried by Stanford groups 4 , but soon it has been shown that it has either the singularity problem or reintroduces a fine-tuning 5 . The solution they obtain has a singularity at say $y=y_{c}$. This is because to cure it, or to satisfy the sum rule, one introduces another brane at $y=y_{c}$. As soon as one introduces a brane, there is one more parameter introduced there, namely the brane tension. A flat space is possible only for a specific value of the new brane tension, needing a fine-tuning. So there does not exists an example for the strong self-tuning solution.

Weak self-tuning solution: This solution does not question whether the flat solution is connected to dS or AdS solutions. Of course, the flat self-tuning solution does not require a fine-tuning(s). It was proposed in early 80's with antisymmetric tensor field strength $H_{\mu \nu \rho \sigma}$. But because that was done in $4 \mathrm{D}$, the idea was not realistic. However, in 5D the weak self-tuning solutions can be made realistic in RS-II models.

In RS-II type models, indeed there exist weak self-tuning solutions 67 . One solution employs the antisymmetric tensor field $A_{M N P}$ in $5 \mathrm{D} A d S_{5}$ and one brane located at $y=0$. The bulk c.c. is $\Lambda_{b}$ and the brane tension is $\Lambda_{1}$. If one introduces the standard term $H^{2}$, there does not exist a self-tuning solution. In fact there exists the no-go theorem with a standard kinetic energy term 8 . The authors of Ref. [5] used $1 / H^{2}$ term where $H^{2}=H_{M N P Q} H^{M N P Q}$, and found a self-tuning solution. The action is

$$
S=\int d^{4} x d y\left\{\frac{1}{2} R_{(5)}-\frac{2 \cdot 4 !}{H^{2}}-\Lambda_{b}-\Lambda_{1} \delta(y)\right\}
$$

where $R_{(5)}$ is the $5 \mathrm{D}$ Ricci scalar and we set the $5 \mathrm{D}$ fundamental mass $M=1 . \Lambda_{b}$ and $\Lambda_{1}$ define two mass parameters: $k_{b}=\sqrt{-\Lambda_{b} / 6}, k_{1}=\Lambda_{1} / 6$. The self-tuning solution with the $Z_{2}$ symmetry condition is given by

$$
\beta(y)=\left[\frac{a}{k_{b}} \cosh \left(4 k_{b}|y|+c\right)\right]^{-1 / 4}
$$

where $a$ is an integration constant from the field equation of $A_{M N P}$ and $c$ is another integration constant. Note that the brane tension $\Lambda_{1}$ is not fine-tuned. But as we will discuss, it must be bounded for the self-tuning solution to be possible. $a$ is determined by the magnitude of the 4D Planck mass, and $c$ is determined by the boundary condition at the brane $-\left.\beta(y)^{\prime}\right|_{y=0^{+}}=\Lambda_{1} / 6$ where $\beta$ is normalized as $\beta(0)=1$. This self-tuning solution is connected to dS and AdS solutions 6 , hence it is a weak self-tuning solution. Since the solution is given in a closed form, it can help studying some properties of weak self-tuning solutions. The existence of the self-tuning solution in the RS-II setup can be recognized by looking at the equation

$$
\left|\beta^{\prime}\right|=\sqrt{\bar{k}^{2}+k_{b}^{2} \beta^{2}-a^{2} \beta^{10}},
$$


where $\bar{k}$ is the effective curvature of the $4 \mathrm{D}$ space, $\bar{k}^{2}=+, 0,-$, corresponding to $\mathrm{dS}$, flat and AdS, respectively. Note that the positive power of $\beta$ in the $a^{2}$ term in Eq. (7) when one uses $1 / H^{2}$. It would have a negative power $\beta^{-6}$ if one used $H^{2}$ instead. The flat solution needs $\beta^{\prime} \rightarrow 0$ as $\beta \rightarrow 0$. Therefore, the case $H^{2}$ does not satisfy this self-tuning solution criterion. On the other hand, $1 / H^{2}$ term satisfies this condition. From this observation, one can find more self-tuning solutions 7 . Of particular interest among these is the self-tuning solution from the log function $\log \left(H^{2}\right)$. Since the solution with $1 / H^{2}$ is given already, in the remainder of this talk we focus on this solution given in (6). The boundary condition at $y=0$ determines $c$

$$
\tanh (c)=\frac{\Lambda_{1}}{\sqrt{-6 \Lambda_{b}}}
$$

\section{Blowing up solutions}

The condition for the self-tuning solution, Eq. (8), is not always satisfied. It is because the region of tanh is limited to $[-1,+1]$. To see when it is not satisfied, let us note that the boundary condition at $y=0^{+}$requires

$$
\left|\beta^{\prime}\right|_{0^{+}}=\sqrt{\bar{k}^{2}+k_{b}^{2}-a^{2}}=\frac{\Lambda_{1}}{6}
$$

where $k_{b}^{2}=\frac{-\Lambda_{b}}{6}$. Therefore, the condition that the flat solution is not allowed is

$$
\frac{\left|\Lambda_{1}\right|}{6}>\sqrt{\frac{-\Lambda_{b}}{6}}
$$

or

$$
\bar{k}^{2}>a^{2} \longrightarrow \bar{\Lambda}>6 a^{2} M^{2} \beta^{10}(0)
$$

where $\bar{\Lambda}$ is the effective curvature of the $4 \mathrm{D}$ space. Thus, only the de Sitter space is allowed where the flat space solution is forbidden 9. This situation is shown in Fig. 1 (b) as the shaded regions. The light(dark) shaded region is where $\Lambda_{1}$ is positive(negative). For comparison, in Fig. 1 (a), we show the $4 \mathrm{D}$ case where the flat space is possible only for $\Lambda_{\text {eff }}=0$. This $\Lambda_{\text {eff }}=0$ is blowed up to a finite region, $-\sqrt{-6 \Lambda_{b}}<\Lambda_{1}<+\sqrt{-6 \Lambda_{b}}$, in Fig. 1 (b). Note that the AdS solution is also forbidden where the flat space solution is forbidden.

The region of the dS-only region is distinguished by positive and negative tensions. Because the negative tension leads to a phantom with negative kinetic energy 10], we adopt the parameters such that only a positive tension results.

This behavior of blowing up is a desirable property of weak self-tuning solutions. For the $1 / H^{2}$, it was possible to show the blowing-up property as given above but it is difficult to see it if one does not have a closed form solution. 


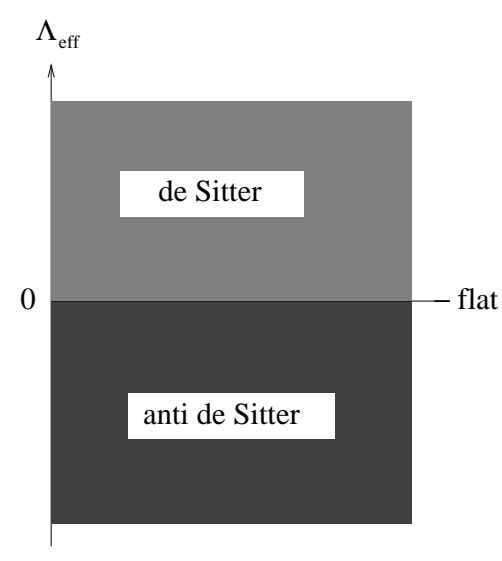

(a)

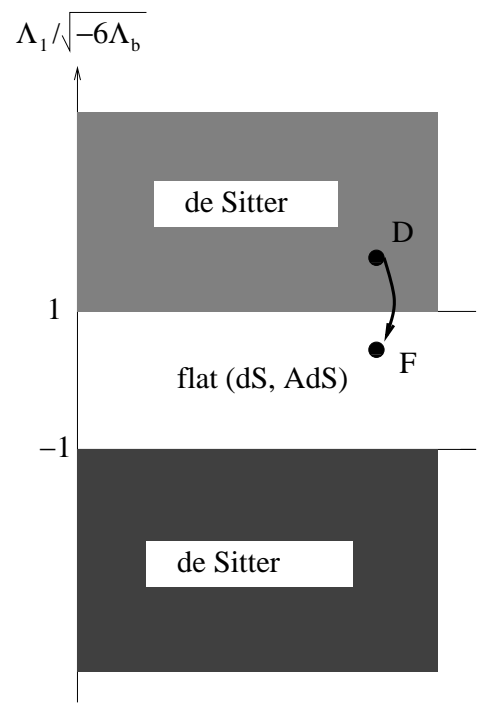

(b)

Fig. 1. A schematic illustration of the blow-up solution. The universe at the dS phase(Point D) goes into the flat-allowed region(Point $\mathrm{F}$ ) by the parameter change at the brane.

\section{Inflation with self-tuning solution}

If only the de Sitter space is allowed for a finite range of parameter space in the Lagrangian, it is tempting to use it for a condition for inflation. By dynamics at the brane, we propose that after a sufficient inflation the parameter settles to the region where flat space solutions are possible. If this happens, say the universe goes from D to F in Fig. 1 (b), the universe at F starts from a de Sitter space solution. It seems to be possible because a sudden change of the $\Lambda_{1}$ parameter would not change the effective c.c. abruptly. But the $\mathrm{F}$ region also allows the flat space, and the key question is how the flat space is chosen from a dS solution after the sudden change of $\Lambda_{1}$. We hope to find a reasonable history for this scenario, which unifies the solutions of the large c.c.(for GUT phase inflation) and the vanishing c.c.(by the self-tuning solution). This is the dream we hoped to understand inflation on top of the vanishing cosmological constant from the time of the birth of inflation 11.

For this purpose, let us adopt the hybrid inflation at the brane. The key point of the hybrid inflation is to use multi fields among which there are an inflaton field $\phi$ and a waterfall field $\psi$ 12. This setup is particularly relevant for our scenario since at the brane $\Lambda_{1}$ can change instantaneously. The coupled potential of the fields $\phi, \psi$ living on the brane is taken as

$$
V=V_{0}+\frac{1}{2} m^{2} \phi^{2}+\frac{1}{2}\left(-m_{\psi}^{2}+\lambda^{\prime} \phi^{2}\right) \psi^{2}+\frac{1}{2} \lambda^{\prime} \psi^{2} \phi^{2}+\Lambda_{1}
$$

where $V_{0}=m_{\psi}^{2} \mu^{2}, m_{\psi}^{2}=\lambda \mu^{2}$, and we treat $\mu^{2}$ and $m^{2}$ the large and small parame- 
ters, respectively. For $\phi$ greater than the critical value $\phi_{c}=\mu \sqrt{\lambda / \lambda^{\prime}}$, the waterfall field is kept at origin $\psi=0$, and there results a slow roll inflation. Requiring $m^{2}$ to be smaller than the Hubble parameter, $m^{2} \ll H^{2}$, we obtain

$$
\mu^{2} \gg \sqrt{\frac{12}{\lambda}} m M_{P l}
$$

where $M_{P l}=1.2 \times 10^{19} \mathrm{GeV}$. The condition (13) also guarantees a sufficient inflation. The condition for forbidding a flat space solution is

$$
\mu^{4}>\sqrt{\frac{96}{\xi \lambda^{2}} M_{P l}^{2} \sqrt{a k_{b}}\left|\Lambda_{b}\right|}
$$

where $\xi=O(1)$. For example, the eyeball numbers, $M=8.4 \times 10^{16} \mathrm{GeV}, \mu>$ $2.2 \times 10^{15} \mathrm{GeV}, V_{0} \sim \bar{\Lambda}>\left(1.86 \times 10^{13} \mathrm{GeV}\right)^{4}$, satisfy these conditions. Thus, we can obtain reasonable numbers from GUT related models. If $\phi$ crosses the critical value $\phi_{c}$, the origin of $\psi$ becomes the vacuum for tachyonic $\psi$, and the waterfall field $\psi$ runs into the true vacuum immediately 12 . This parameter change occurs at the brane, and the brane tension after the waterfall field settles at the minimum is $\Lambda_{1}$. Namely, the universe settles at the point $F$, with a nonzero brane tension $\Lambda_{1}>0$, after the waterfall field finds the minimum. It has been shown that the parameters of the above hybrid inflation can fall in the region which GUT phase transitions allow 9 .

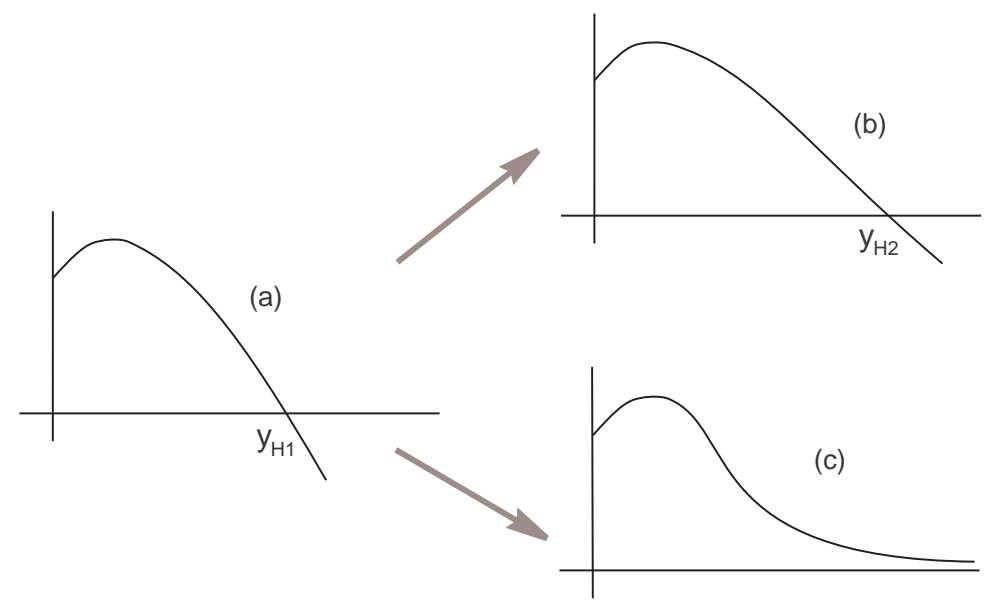

Fig. 2. A schematic illustration of the transition from a dS space with horizon $H 1$ (a) to another dS space with horizon $H_{2}$ (b), or to a flat space (c).

Immediately after the hybrid inflation at the brane, our self-tuning solution is expected to be a dS one in the flat allowed blowing-up region. Then, one can imagine that the $\mathrm{dS}$ solution undergoes to a solution with a time-dependent c.c. 
But the a closed form dS solution is not obtained. At the moment, the best we can obtain is the existence of the time-dependent c.c. For this, we studied a step function change of c.c. with a metric with time-dependent $b(t)$ 13. As illustrated in Fig. 2, one anticipates the solution with the curvature change, i.e. the horizon distance changes from $y_{H 1}$ (a) to $y_{H 2}$ (b), or to $\infty$ (c). We found that in general there exist solutions for any $y_{H 2}$. Therefore, classical physics does not determine the path. Here, Hawking's probabilistic interpretation 14 is applicable. In our setup, the initial condition after inflation is well defined, i.e. Point F in Fig. 1 (b). Then, it is shown that the probability to choose the flat space, i.e. Fig. 2 (c), is infinitely larger than choosing any other space 14 .

\section{Conclusion}

In conclusion,

(i) We tried to unify two vacuum energy problems, one the sufficient inflation and the other the vanishing cosmological constant.

(ii) A weak self-tuning solution was used for this scenario for the ultimately vanishing cosmological constant. It is possible for some range of inflaton field parameters for inflation to happen, $\Lambda_{1}>\sqrt{-\Lambda_{b} / 6}$.

(iii) For inflation at the brane and the exit from inflation, we showed the possibility in the hybrid inflation model.

(iv) The choice of the flat space after inflation is hoped to be solved classically. If classical physics does not determine the path, quantum corrections must choose the ultimate late universe.

\section{Acknowledgments}

This work is supported in part by the KOSEF ABRL Grant No. R14-2003-01201001-0, the BK21 program of Ministry of Education, and Korea Research Foundation Grant No. KRF-PBRG-2002-070-C00022.

\section{References}

1. C. Wetterich, Nucl. Phys. B302, 668 (1988); P. J. E. Peebles and B. Ratra, Astrophys. J. 325, L17 (1988); J. A. Frieman, C. T. Hill and R. Watkins, Phys. Rev. D46, 1226 (1992); C. Kolda and D. H. Lyth, Phys. Lett. B458, 197 (1999); J. E. Kim, JHEP 9905, 022 (1999); J. E. Kim and H. P. Nilles, Phys. Lett. B553, 1 (2003).

2. M. Veltman, Phys. Rev. Lett. 34, 777 (1975).

3. L. Randall and R. Sundrum, Phys. Rev. Lett. 83, 4690 (1999). A precursor is, V. A. Rubakov and M. E. Shaposhnikov, Phys. Lett. B125, 136 (1983).

4. S. Kachru, M. B. Schulz, and E. Silverstein, Phys. Rev. D62, 045021 (2000); N. ArkaniHamed, S. Dimopoulos, N. Kaloper, and R. Sundrum, Phys. Lett. B480, 193 (2000).

5. S. Förste, Z. Lalak, S. Lavignac, and H. P. Nilles, Phys. Lett. B481, 360 (2000); JHEP 0009, 034 (2000). 
8 Jihn E. Kim

6. J. E. Kim, B. Kyae, and H. M. Lee, Phys. Rev. Lett. 86, 4223 (2001); Nucl. Phys. B613, 306 (2001); K.-S. Choi, J. E. Kim, and H. M. Lee, J. Kor. Phys. Soc. 40, 207 (2002).

7. J. E. Kim and H. M. Lee, JHEP 0209, 052 (2002).

8. C. Csaki, J. Erlich, C. Grojean, and T. Hollowood, Nucl. Phys. B584, 359 (2000).

9. J. E. Kim, JHEP 0301, 042 (2003).

10. J. Garriga and T. Tanaka, Phys. Rev. Lett. 84, 2778 (2000).

11. A. Guth, Phys. Rev. D23, 347 (1981).

12. A. D. Linde, Phys. Lett. B249, 18 (1990); F. C. Adams and K. Freese, Phys. Rev. D43, 353 (1991); A. D. Linde, Phys. Lett. B259, 38 (1991).

13. J. E. Kim and H. M. Lee, hep-th/0309046

14. S. W. Hawking, Phys. Lett. B134, 403 (1984). 